\title{
Death, taxes, and relentless evolution in nature
}

\author{
Relentless Evolution by John N. Thompson, University of Chicago Press, 2013. US\$35.00/£22.50, pbk (499 pp.) ISBN 978-0-226-01875-1
}

\section{Jonathan L. Richardson}

Department of Ecology and Evolutionary Biology, University of Connecticut, 75 N. Eagleville Road, Storrs, CT 06269-3043, USA

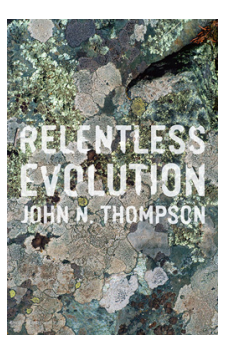

Over the past 15 years, there has been a surge of interest in evolution occurring over short time spans. Evolution identified as rapid or, less ambiguously, as contemporary with ecological processes has received theoretical support and been observed in an increasing number of species as researchers focus on the temporal aspects of adaptation in more and more systems. Researchers have made significant strides in formalizing the concept and quantifying its occurrence in nature [1-3]. John Thompson was one of the key catalysts of this swell in interest in contemporary evolution with his 1998 perspective article [4].

In his new book Relentless Evolution, Thompson takes on the immense challenge of synthesizing not only the body of literature that has accumulated over the past 15 years, but also the tangential aspects of evolution and ecology that are instrumental in appreciating the persistence of evolutionary dynamics and the surprising speed of evolution. The book is organized according to the complexity of the evolving units, starting with genes and ending with the evolution of species interactions in the web of life. Along the way are relevant chapters on natural selection, hybridization, experimental evolution, and ecological speciation.

Each chapter begins with a relevant background discussion and contains many illustrative examples from the literature. It is clear throughout that Thompson has a special mastery of the literature, incorporating the historical foundations of these topics in evolutionary biology as well as many pertinent studies from the past 5 years. The comprehensive overview of the literature makes this book a valuable and accessible resource for anyone with a basic interest in evolution and ecology, including graduate students, faculty, and engaged non-scientists.

The most salient message of the book is critically important for biology: environments are always changing and species and populations are always responding to these fluctuations via evolutionary change. As a result, it is unreasonable to treat stasis in natural systems as the null hypothesis. Thompson uses the metaphor of populations as moored boats sitting in the water, constantly rising and falling with the tides and storms. Some populations may be better at adjusting to changes, but most are able to stay afloat because of sufficient levels of genetic and trait variation. In this case, the adaptive landscape is better visualized as turbulent water rather than stable peaks and valleys. Thompson argues effectively that

Corresponding author: Richardson, J.L. (jrichardson@uconn.edu). the myopic focus on sustained directional selection by evolutionary ecologists misses the more common (and dynamic) meanderings of fluctuating evolution in response to variable selection.

Although Thompson highlights the relentlessness of evolution over time, how persistent is evolution across space? Refreshingly, he devotes some space in the chapter on local adaptation to the spatial scale of adaptive divergence observed in nature. This explicit treatment of space is often lacking in evolutionary ecology, replaced instead with acknowledgments of the role of gene flow in shaping adaptation. But here, Thompson misses the opportunity to move beyond the subjective criteria regarding the scale of absolute distances at which local adaptation is unexpected or surprising. This spatial scale is entirely relative to the study taxon being considered. Thompson uses the same criteria of absolute distances between diverged groups that seem surprising (tens of meters for pathogen resistance to hundreds of kilometers in marine invertebrates) to frame his discussion of local adaptation at small scales. This is the current state of thinking on the geographic scale of evolution, one that we need to advance using standardized measures of the spatial extent of adaptation scaled to dispersal abilities.

Perhaps representing the lack of consensus across biology, Thompson also becomes overly vague when defining species in the chapter on ecological speciation, describing them as "clouds of individuals whose fitnesses in various environments are distributed differently from other species.' However, he follows this up with an excellent overview of the current state of thinking on ecological speciation. This includes the recent shift away from inherent 'genetic incompatibilities' as the primary driver of reproductive isolation toward an appreciation of the role of 'ecological incompatibilities' between locally adapted groups.

Environments will continue to experience inexorable changes driven by natural fluctuations and shifting global climates. In turn, species and populations will continue to evolve in response. This relentless evolution will play a critical role in how species respond to changes in future climates - some will be able to respond and persist, whereas others will become unmoored from the dock. The likely extinctions get most of the attention in the literature and media [e.g., 5-7]. However, Thompson's thorough treatment of rapid and persistent evolution will hopefully place a spotlight on the remarkable, but not limitless, ability of nature to mitigate environmental change $[8,9]$. I believe that this appreciation of fluctuating natural selection and the incessant evolutionary responses of populations will be the enduring message 
from Relentless Evolution. Embracing this message will likely lead to a new set of expectations (and important advances) in evolution and ecology where evolutionary change is ubiquitous, occurs quickly, and is underpinned by the substantial levels of trait and genetic variation we see in nature.

\section{References}

1 Kinnison, M.T. and Hendry, A.P. (2001) The pace of modern life II: from rates of contemporary microevolution to pattern and process. Genetica $112,145-164$

2 Hairston, N.G. et al. (2005) Rapid evolution and the convergence of ecological and evolutionary time. Ecol. Lett. 8, 1114-1127

3 Ellner, S.P. et al. (2011) Does rapid evolution matter? Measuring the rate of contemporary evolution and its impacts on ecological dynamics. Ecol. Lett. 14, 603-614
4 Thompson, J.N. (1998) Rapid evolution as an ecological process. Trends Ecol. Evol. 13, 329-332

5 Thomas, C.D. et al. (2004) Extinction risk from climate change. Nature 427, $145-148$

6 Wake, D.B. and Vredenburg, V.T. (2008) Colloquium paper: are we in the midst of the sixth mass extinction? A view from the world of amphibians. Proc. Natl. Acad. Sci. U.S.A. 105, 11466-11473

7 Urban, M.C. et al. (2012) On a collision course: competition and dispersal differences create no-analogue communities and cause extinctions during climate change. Proc. R. Soc. B 279, 2072-2080

8 Gienapp, P. et al. (2008) Climate change and evolution: disentangling environmental and genetic responses. Mol. Ecol. 17, 167-178

9 Hoffmann, A.A. and Sgrò, C.M. (2011) Climate change and evolutionary adaptation. Nature 470, 479-485

0169-5347/\$ - see front matter

http://dx.doi.org/10.1016/j.tree.2013.09.014 Trends in Ecology \& Evolution xx (2013) 1-2 